\title{
Expression of IncRNAs in children with pancreaticobiliary maljunction: functional analysis and potential biomarkers
}

\author{
Lian Zhao ${ }^{1}$, San-Li Shi², Wan-Liang Guo ${ }^{1}$
}

${ }^{1}$ Children's Hospital of Soochow University, China ${ }^{2} 8^{\text {th }}$ Hospital of Xi'an, China

Submitted: 9 October 2021; Accepted: 2 January 2022

Online publication: 6 January 2022

Arch Med Sci

DOI: https://doi.org/10.5114/aoms/145482

Copyright $\odot 2022$ Termedia \& Banach

\section{Abstract}

Introduction: Pancreaticobiliary maljunction (PBM) leads to higher rates of complications, including cholangitis, pancreatitis, and malignancies. The aim of the present study was to investigate the expression profile of long non-coding RNAs (IncRNAs) and their potential role as biomarkers in children with pancreaticobiliary maljunction.

Material and methods: The differential expression of IncRNAs and messenger RNA (mRNAs) from pediatric patients with pancreaticobiliary maljunction and control subjects was analyzed using a commercial microarray and later validated with qRT-PCR. The potential biological functions of differentially expressed genes were explored based on Gene Ontology and Kyoto Encyclopedia of Genes and Genomes pathway enrichment. The ability of potential IncRNA biomarkers to predict pancreaticobiliary maljunction was assessed based on the area under the receiver operating characteristic curve (AUC).

Results: There were 2915 mRNAs and 173 IncRNAs upregulated, and 2121 mRNAs and 316 IncRNAs downregulated in PBM cases compared to controls. The enriched Gene Ontology categories associated with differentially expressed mRNAs were extracellular matrix, extracellular region, and kinetochore. The most enriched Kyoto Encyclopedia pathway was protein digestion and absorption, which was associated with cancer and PI3KAkt signaling. Analysis of cis- and trans-target genes predicted that a single IncRNA was able to regulate several mRNAs. The qRT-PCR results for $N R \_110876, N R \_132344, X R \_946886$, and XR_002956345 were consistent with the microarray results, and the difference was statistically significant for NR_132344, XR_946886, and XR_002956345 ( $p<0.05)$. AUC was significant only for $X R_{-} 946886(0.837, p<0.001)$.

Conclusions: Our results implicate IncRNAs in common bile duct pathogenesis in PBM, and they identify XR_946886 as a potential biomarker for the disease.

Key words: pancreaticobiliary maljunction, long non-coding RNA, gene ontology, biomarker, differentially expressed genes.

\section{Introduction}

Pancreaticobiliary maljunction (PBM) is a rare congenital anomaly in which the junction of the pancreatic and biliary ducts is located outside the duodenal wall, with a long common channel [1-3]. The resulting twoway reflux of bile and pancreatic juice leads to several complications,

\author{
Corresponding author: \\ Wan-Liang Guo \\ Children's Hospital \\ of Soochow University \\ 303\#, Jingde Road \\ 215003, Suzhou, China \\ Phone: +8615950011521 \\ E-mail: gwlsuzhou@163.com
}


including bile retention with cholangitis, pancreatitis, and malignancies [4-6].

Cyst excision and Roux-en-Y hepaticojejunostomy are the first choice for PBM treatment [7-9]. However, these procedures are associated with postoperative complications affecting the residual bile ducts, such as cholangitis, hepatolithiasis, and carcinogenesis [8, 10-13]. Identification of biomarkers associated with PBM complications of the bile duct could enable the early detection of these complications and support the development of effective diagnostic and therapeutic methods.

Long noncoding RNAs (IncRNAs), which are non-coding RNAs longer than 200 nucleotides, play an important role in regulating the occurrence and progression of many diseases, such as infectious and malignant tumors [14-16]. They exert these effects by modulating biological processes such as cellular proliferation, motility, and immune response to diseases [17-19]. In addition, some IncRNAs have been identified as biomarkers for diagnosis and prognosis of diseases [20-22]. However, the genome-wide expression and functional roles of IncRNAs in PBM are unclear.

To the best of our knowledge, this is the first study focused on IncRNAs in the common bile duct of PBM. Here, we investigated the expression profile of IncRNAs in the common bile duct of patients with PBM, and we identified IncRNAs differentially expressed relative to controls. We explored the potential functions of the differentially expressed IncRNAs and assessed their ability to serve as biomarkers.

\section{Material and methods}

\section{Material}

The study protocol was approved by the Ethics Committee of our hospital and complied with the Helsinki Declaration. Written informed consent was obtained from the guardian of each subject before surgery. A total of 15 pediatric subjects with PBM and 15 control subjects were included in the study between January 2017 and September 2020. In all patients, diagnosis was confirmed by imaging and surgical pathological examination. PBM was diagnosed based on the following criteria: (1) the union of the pancreatic and biliary duct was located outside the sphincter of Oddi, based on magnetic resonance cholangiopancreatography (MRCP) or intraoperative cholangiography (IOC); (2) the common duct was longer than $5 \mathrm{~mm}$; (3) the biliary amylase level was greater than $1000 \mathrm{U} / \mathrm{I}$ [23].

\section{RNA microarray analysis}

Microarray experiments to investigate IncRNAs and messenger RNA (mRNAs) differentially expressed between the PBM and control groups were performed by Western SCI Biotech Company (Chongqing, China, www.westernsci.cn). Agilent Human IncRNA Microarray 2018 Version (4*180k, Design ID: 085630), Agilent Technologies, Inc., was used.

The common bile duct tissue from PBM subjects was collected and stored at $-80^{\circ} \mathrm{C}$ before RNA extraction. Total RNA was isolated using TRIZOL (Invitrogen, Carlsbad, CA, USA) according to the manufacturer's protocol and quantified using the NanoDrop ND-2000 (Thermo Scientific). RNA integrity was assessed using the Agilent Bioanalyzer 2100. Sample labeling, microarray hybridization, and washing were performed based on the manufacturer's standard protocols. Briefly, total RNA was transcribed to double-stranded cDNA, then synthesized into cRNA and labeled with cyanine-3-CTP. The labeled cRNAs were then hybridized onto the microarray. After washing, the arrays were scanned using the Agilent Scanner G2505C (Agilent Technologies).

Array images were analyzed using Feature Extraction software (version 10.7.1.1, Agilent Technologies), and the raw data were further processed using Genespring (version 13.1, Agilent Technologies). First, the raw data were normalized using the quantile algorithm. The probes that had flags in "P" in at least 1 out of 2 conditions were chosen for further analysis. Differentially expressed mRNAs and IncRNAs were defined as those whose expression differed by $\geq 2$.0-fold between PBM and control groups $(p<0.05)$. The potential roles of these differentially expressed $m R$ NAs and IncRNAs were explored based on Gene Ontology (GO) and Kyoto Encyclopedia of Genes and Genomes (KEGG) pathways (see section 2.3). Finally, the differentially expressed genes were organized using hierarchical clustering.

\section{Prediction of IncRNA function}

Following the approach in a previous study [24], we examined differentially expressed mRNAs for enrichment of KEGG functional pathways, then we attributed the same functional pathways to IncRNAs that were co-expressed with those mRNAs. Co-expression of mRNAs and IncRNAs was defined as a Pearson correlation $p$-value $<0.05$.

The enrichment of functional terms was analyzed using a hypergeometric cumulative distribution function, and the 200 and 500 most reliable predicted relationships were displayed in order to understand the distribution of functions of differentially expressed IncRNAs.

\section{Fine mapping of co-expression of IncRNAs and adjacent coding genes}

For each differentially expressed IncRNA, associated "cis-regulated mRNAs" were identified based 
on the following criteria: (1) the loci encoding the mRNAs were within $300 \mathrm{~kb}$ up- or downstream of the given IncRNA; and (2) the Pearson correlation coefficient for IncRNA-mRNA co-expression was significant $(p \leq 0.05)$. The co-expressed genes and enrichment significance for differentially expressed genes in each transcription factor (TF) entry were calculated for each differentially expressed IncRNA, using the hypergeometric distribution test. The calculated results return a $p$-value of enrichment significance, where a small $p$-value indicates that the gene is enriched.

The intersection between the coding gene set co-expressed by IncRNAs and the target gene set of the TF/chromatin regulatory complex was calculated, and the enrichment degree of the intersection was calculated using a hypergeometric distribution test. This calculation identified the TFs significantly related to differentially expressed IncRNAs, and thereby the TFs and chromatin regulatory factors that may regulate those IncRNAs. The network diagram was visualized based on the results of the hypergeometric distribution analysis.

According to hypergeometric distribution calculations, multiple IncRNA-TF pairs were obtained for each differentially expressed IncRNA. Each IncRNA-TF pair was the result of multiple gene enrichment.

\section{Quantitative reverse transcription- polymerase chain reaction (qRT-PCR) assay}

In order to verify the microarray results, we randomly selected six differentially expressed IncRNAs that were associated with carcinogenesis or chronic inflammation in the common bile duct. We measured the IncRNA expression levels of these genes using qRT-PCR and the SYBR Green PCR Kit (Applied $\mathrm{BI}$ ) according to the manufacturer's instructions. Expression levels were calculated using the $2^{-\Delta \Delta} \mathrm{Ct}$ method and normalized to those of $\beta$-actin IncRNA.

\section{Statistical analysis}

SPSS 20.0 software (IBM, Armonk, NY, USA) was used for statistical analysis. Data were expressed as mean \pm standard deviation (SD), and inter-group differences were assessed for significance using the rank sum test. The diagnostic accuracy of certain differentially expressed IncRNAs was assessed in terms of the area under the receiver operating characteristic curve (AUC). Statistical significance was defined as $p<0.05$.

\section{Results}

\section{General information}

Patients' demographic and clinical data are summarized in Table I. There was no signifi- cant difference between the two groups in body weight, age, or sex distribution.

\section{Differential gene expression between PBM and control subjects}

The microarray analysis identified 2915 upregulated mRNAs, 2121 downregulated mRNAs, 173 upregulated IncRNAs, and 316 downregulated IncRNAs in subjects with PBM compared to healthy controls. Principal component analysis of differentially expressed IncRNAs and a heatmap are presented in Figure 1, while the 20 most up- or downregulated IncRNAs are presented in Table II. The most highly upregulated IncRNA was LOC105378608, which showed a 14.39-fold difference from controls.

\section{Functional analysis of differentially expressed genes}

The most enriched GO categories associated with the differentially expressed transcripts were extracellular matrix ( $p=2.9 \mathrm{E}-12$, up-regulated), extracellular region ( $p=0.000427$, up-regulated), and kinetochore ( $p=2.29 \mathrm{E}-8$, up-regulated) (Figure 2). The most enriched KEGG pathway was protein digestion and absorption, in which 250 differentially expressed genes were involved. Some pathways were associated with cancer, such as pathways in cancer (associated with 11 genes) and the PI3K-Akt signaling pathway (associated with 15 genes, Figure 3).

\section{Co-expression analysis and target prediction}

The functions of IncRNAs are performed by interacting with their targets. We predicted both cisand trans-acting mRNAs that may interact with differentially expressed IncRNAs. The cis analysis

Table I. Clinicodemographic characteristics of study subjects

\begin{tabular}{|lcc|}
\hline Characteristic & $\begin{array}{c}\text { Todani types I Todani types IV } \\
(n=7)\end{array}$ & \begin{tabular}{c}
$(n=8)$ \\
\hline Abdominal pain
\end{tabular} \\
\hline Jaundice & 1 & 4 \\
\hline Mass & 0 & 0 \\
\hline Fever & 1 & 1 \\
\hline Vomiting & 5 & 3 \\
\hline Sex (male) & 0 & 3 \\
\hline Age [months] & $9-71$ & $4.5-50$ \\
\hline Pathological findings: & & 8 \\
\hline cyst wall hyperplasia & 7 & 8 \\
\hline gallbladder wall & 7 & - \\
\hline congestion & - & \\
\hline
\end{tabular}



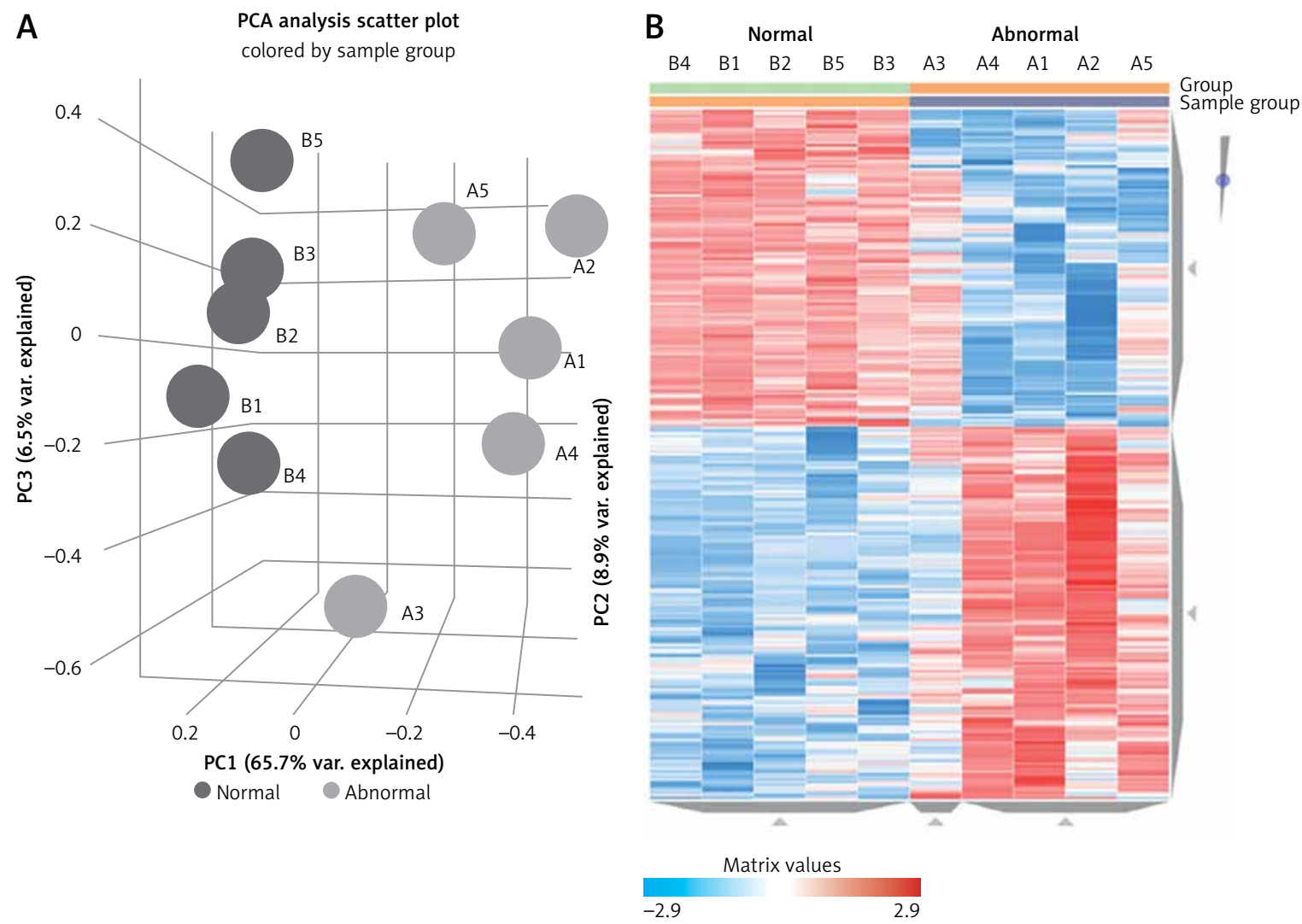

Figure 1. A - Principal component analysis of differentially expressed IncRNAs. Principal component 1 explained $65.7 \%$ of the observed variance; component 2, 8.9\%; and component 3, 6.5\%. B - Heatmap of differentially expressed IncRNAs

searched for mRNAs $300 \mathrm{~kb}$ up- or downstream of the IncRNAs, and it identified 5 IncRNAs related to those cis target genes (Figure 4). The trans analysis of IncRNAs was performed by constructing co-expression networks of dysregulated mRNAs and IncRNAs based on Pearson expression correlation (Figure 5). Most of the differentially expressed IncRNAs acted in a trans manner. The top 200 predicted relationships with the highest prediction reliability were used to determine which TFs occurred most frequently, and the functions of these TFs were then attributed to the differentially expressed IncRNAs (Figure 6). Among the 964 differentially expressed IncRNAs and the predicted 9358 mRNA targets, more than one mRNAs were predicted to be regulated by one IncRNA and one mRNA corresponded to several IncRNAs.

\section{Validation by qRT-PCR}

Six up-regulated IncRNAs associated with carcinogenesis or chronic inflammation in the common bile duct were verified by qRT-PCR: NR_110876, NR_132344, XR_946886, $X R \_002956345, N R \_135295$, and XR_002957935. The expression differences between patients and controls for NR_110876, NR_132344, XR_946886, and XR_002956345 were consistent with the mi- croarray results, and the difference was statistically significant for NR_132344, XR_946886, and $X R \_002956345$. However, the qRT-PCR results for $N R \_135295$ and $X R_{-} 002957935$ were inconsistent with the microarray results (Figure 7).

To assess the potential of NR_110876, $N R \_132344, X R \_946886$, and XR_002956345 as PBM biomarkers, we examined their ability to predict PBM in our sample. Only XR_946886 gave a significant AUC $(0.837, p<0.001)$ (Figure 8).

\section{Discussion}

Here we identified several IncRNAs that are differentially regulated in PBM, and we explored their potential biological functions.

Congenital dilation of the common bile duct has been shown to involve numerous alterations in the transcriptome, and PBM has been associated with downregulation of several genes in the gallbladder, as well as upregulation of some noncoding RNAs, which may contribute to biliary carcinogenesis [25]. In our previous study [26], we found 876 differentially expressed genes in children with PBM, of which 530 were up-regulated and 346 were down-regulated. Wong et al. reported on patients with congenital dilation of the common bile duct, human developmental 


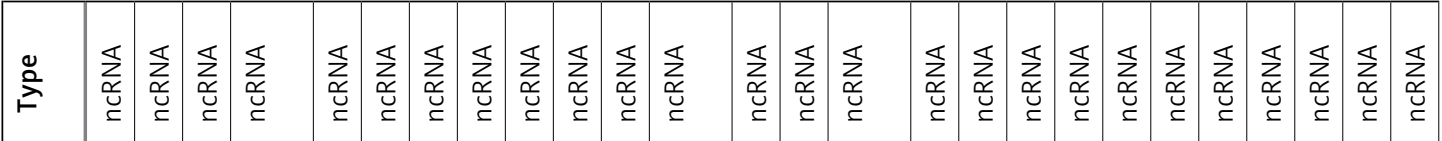

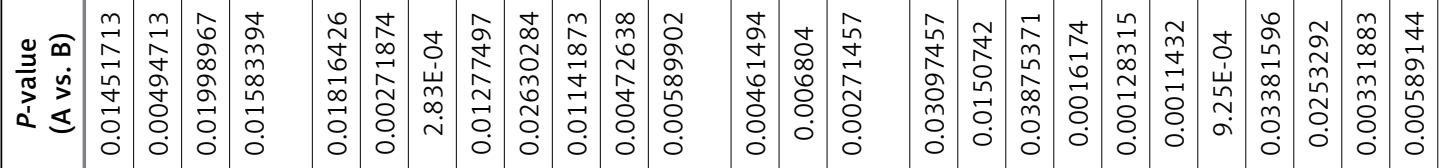

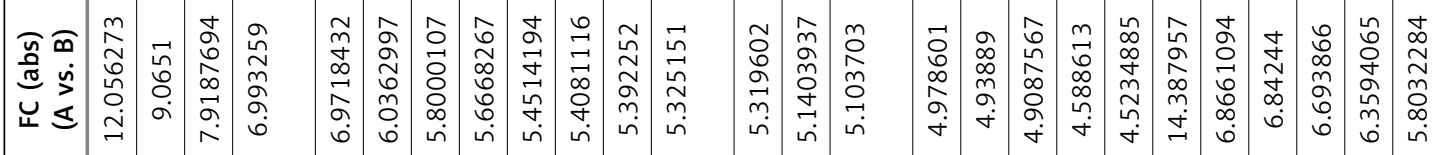

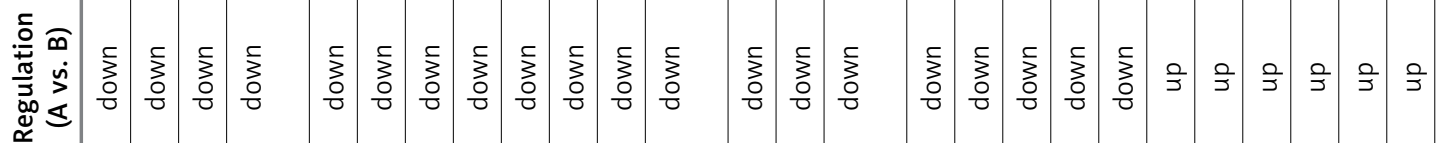

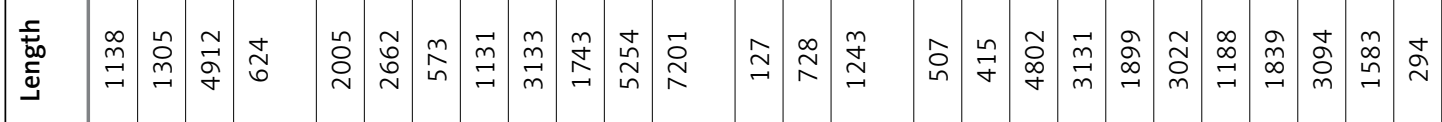

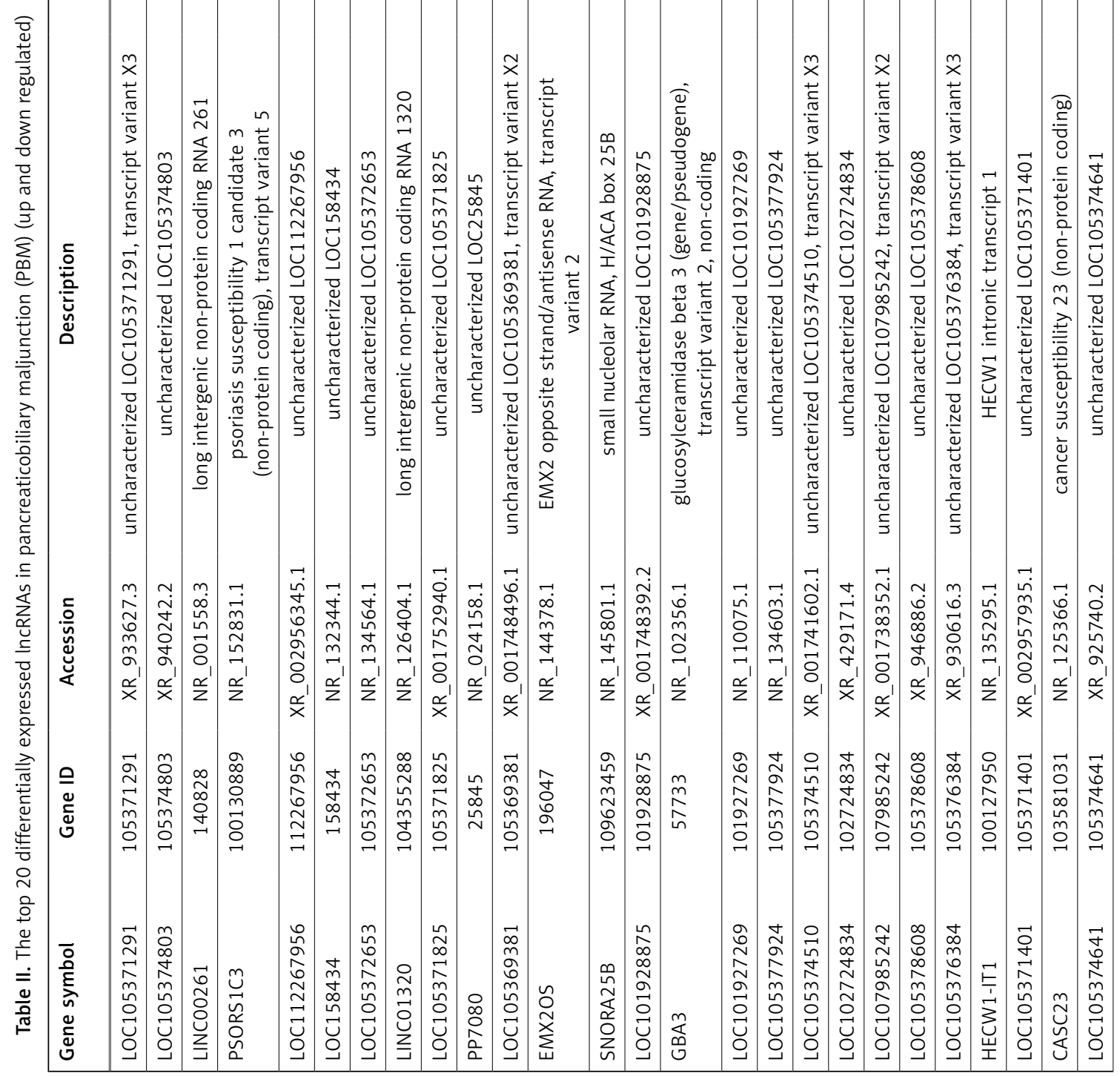




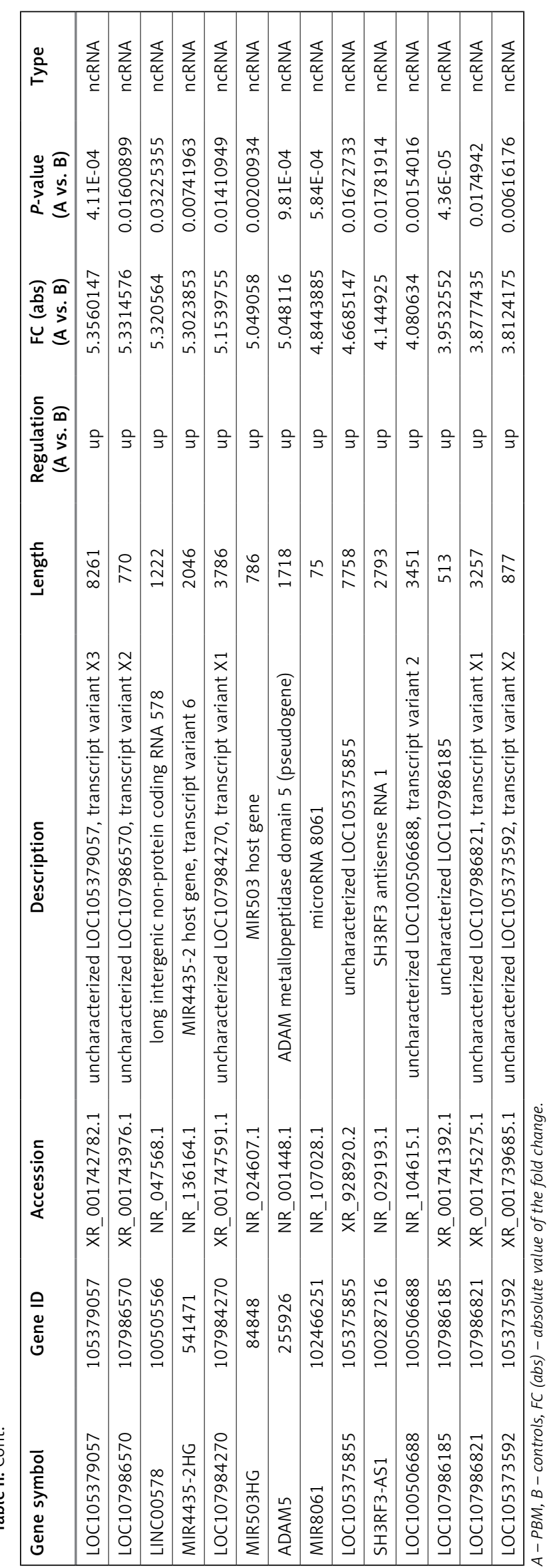

disorders, cholangiocarcinoma or hepatocellular carcinoma [27]. However, we are unaware of investigations of IncRNAs in PBM.

In the present study, we identified 489 IncRNAs and 5036 mRNAs in bile duct tissues that were differentially expressed in PBM. These differentially expressed IncRNAs may affect many pathways, including those involved in protein digestion and absorption, interactions between extracellular matrix and surface receptors, focal adhesion, PI3K-Akt signaling, carcinogenesis, and inflammation. These predictions are consistent with previous studies [22, 24, 25]. In this way, our study implicates IncRNAs in PBM-associated chronic inflammation and carcinogenesis in the common bile duct.

We explored the potential functions of differentially expressed IncRNAs based on the functions of their target genes. We found that 5 cis-target genes were related to differentially expressed IncRNAs, and that most IncRNAs acted in a trans manner. For example, we found that one IncRNA (NR_027038.1) was closely associated with E2F4, which is consistent with previous reports linking E2F4 to development and progression of cancers $[28,29]$.

Of the many IncRNAs that we identified as differentially expressed, validation of a subset using qRT-PCR showed that not all microarray results were accurate. Nevertheless, we were able to validate upregulation of NR_110876, NR_132344, $X R_{-}$946886, and XR_002956345. Among these IncRNAs, XR_946886 showed significant diagnostic potential. Thus, this IncRNA seems likely to be involved in the development of PBM complications, and it may serve as a novel biomarker for carcinogenesis or chronic inflammation involving the common bile duct [30].

This is the first study focused on IncRNAs in the common bile duct of PBM. Our findings may provide a basis for developing novel diagnostic and therapeutic targets in PBM. There were some limitations to the present study. The sample was relatively small, and only six IncRNAs identified in microarray experiments were validated using qRT-PCR. Future studies should be conducted with larger samples and more detailed validation and analysis of candidate genes. Future studies should also explore the potential mechanism of $X R_{-} 946886$ in PBM and its clinical potential as a biomarker.

In summary, the present study provided an overall analysis of IncRNAs in the common bile duct in PBM. By further exploring potential functions and pathways in which differentially expressed IncRNAs are involved, we anticipate that IncRNAs such as XR 946886 will be validated as biomarkers or even potential therapeutic targets for PBM treatment. 
A

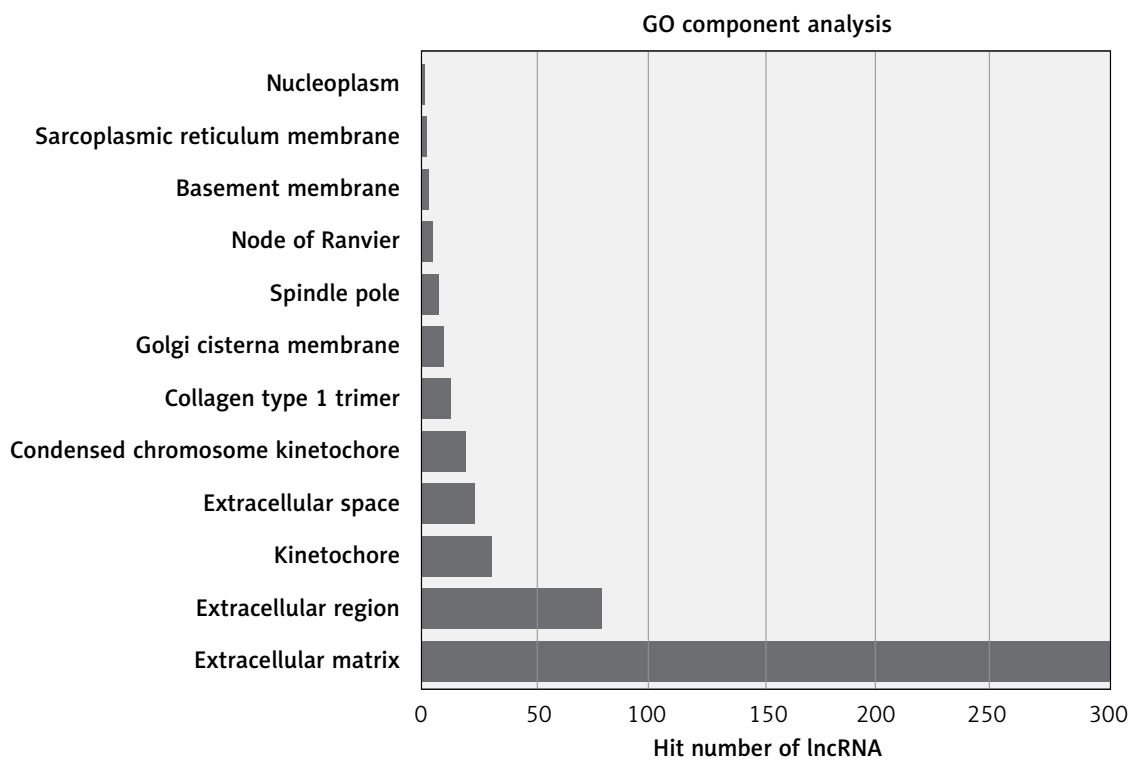

B

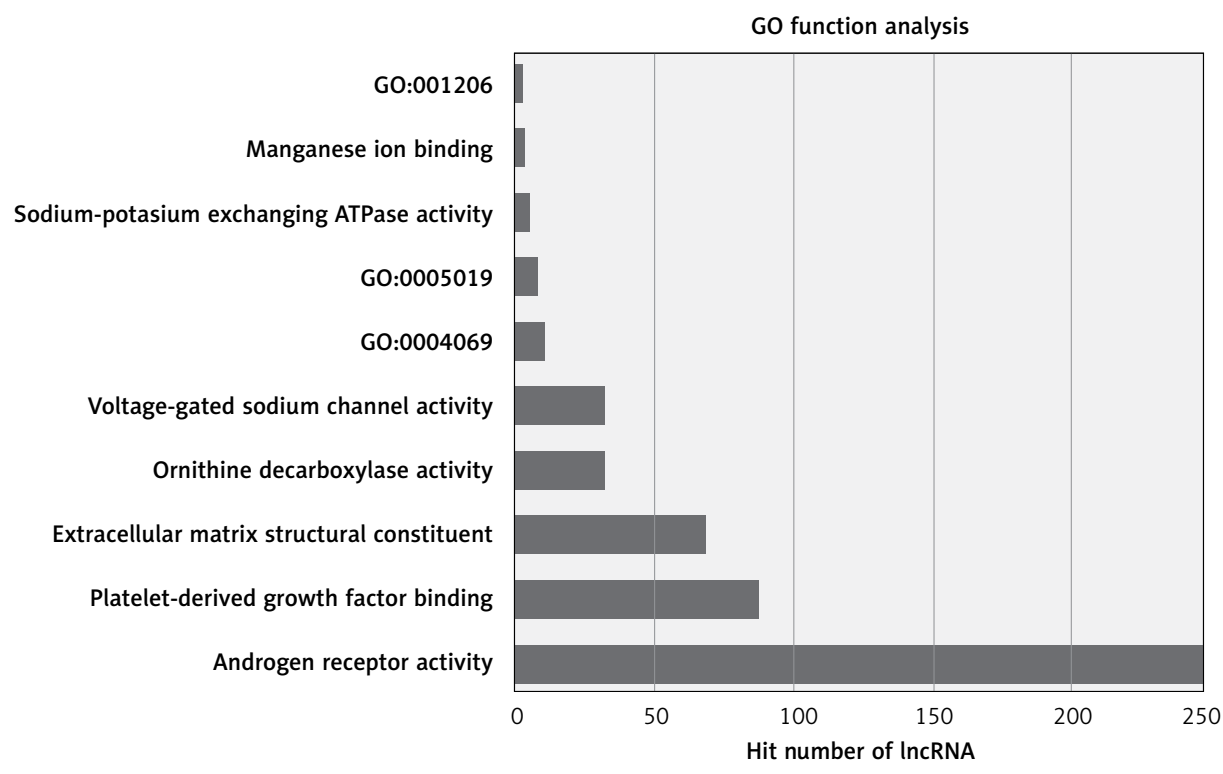

C

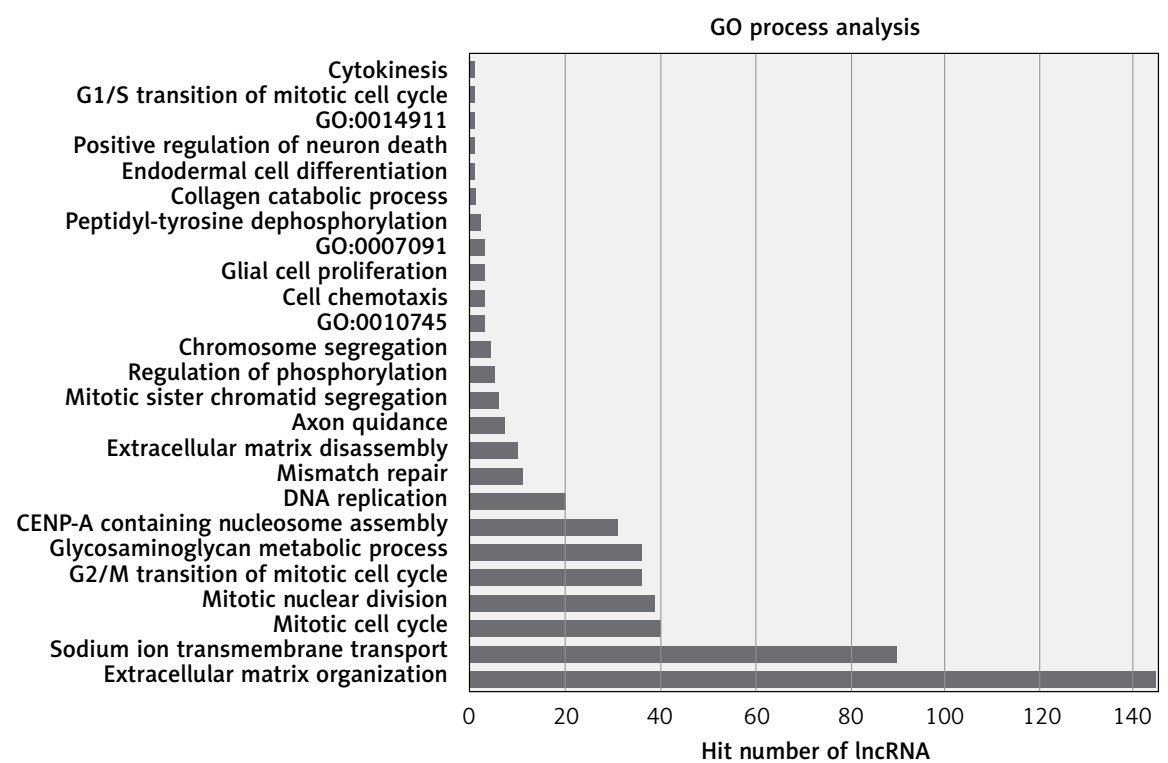

Figure 2. Functional analysis of differentially expressed genes based on Gene Ontology (GO) enrichment analysis 


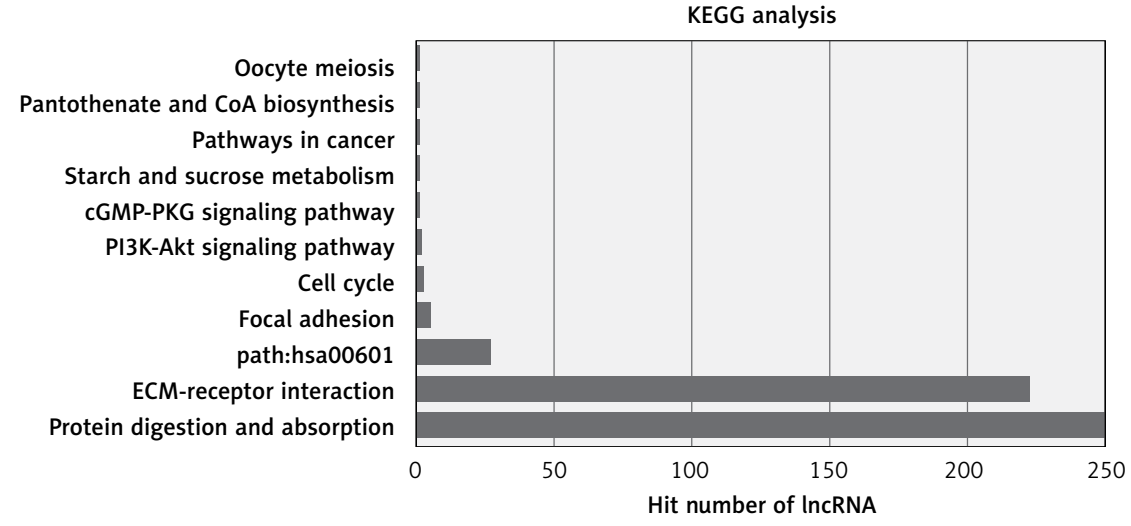

Figure 3. Analysis of Kyoto Encyclopedia of Genes and Genomes (KEGG) pathway enrichment in differentially expressed IncRNAs

A

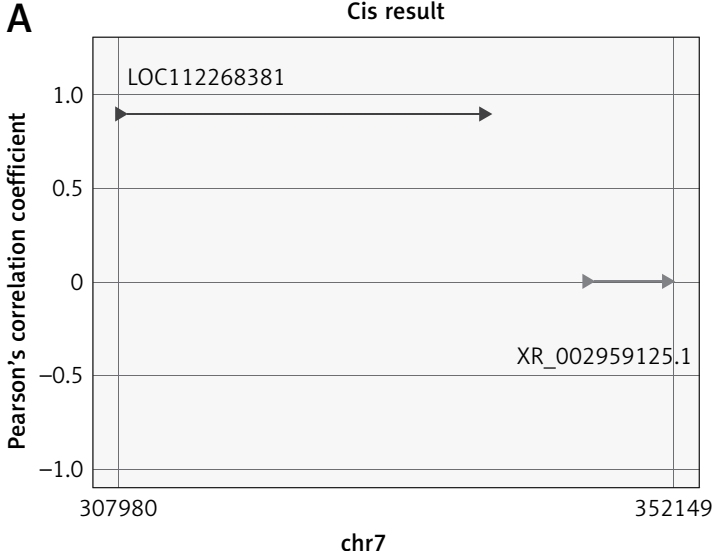

C

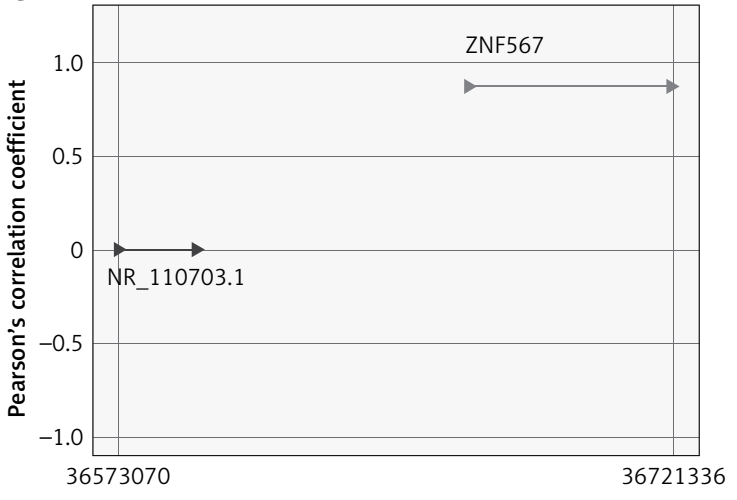

chr19

E

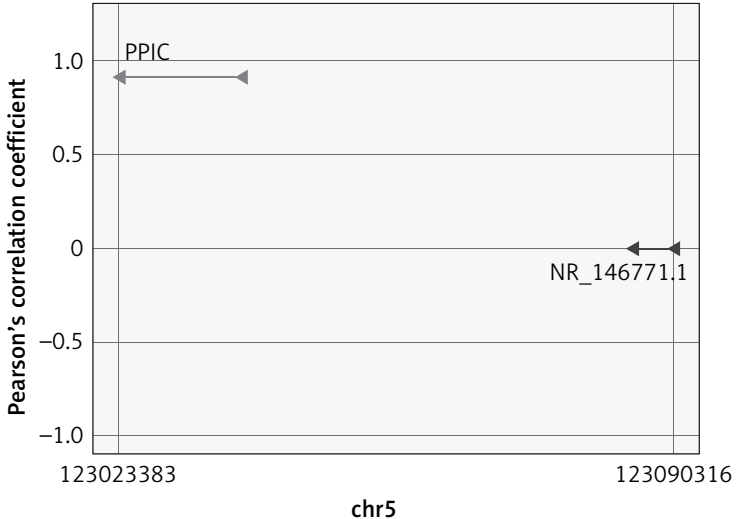

B Cis result

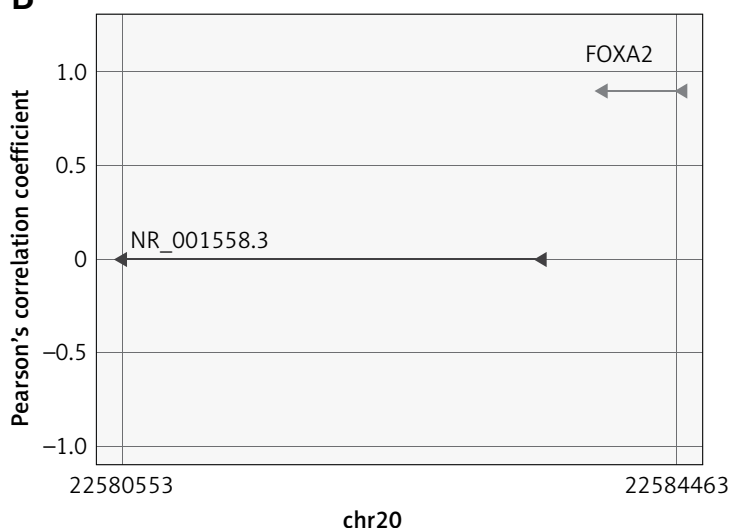

D

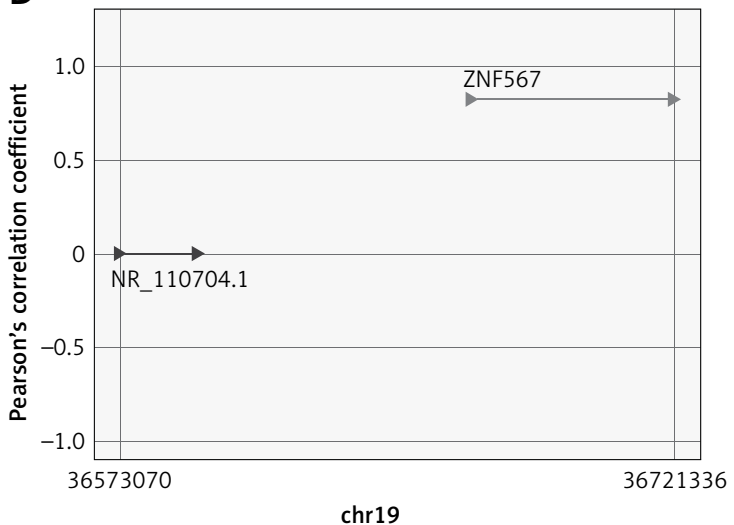

Figure 4. Association of five differentially expressed IncRNAs with cis-target genes in pancreaticobiliary maljunction (PBM) 


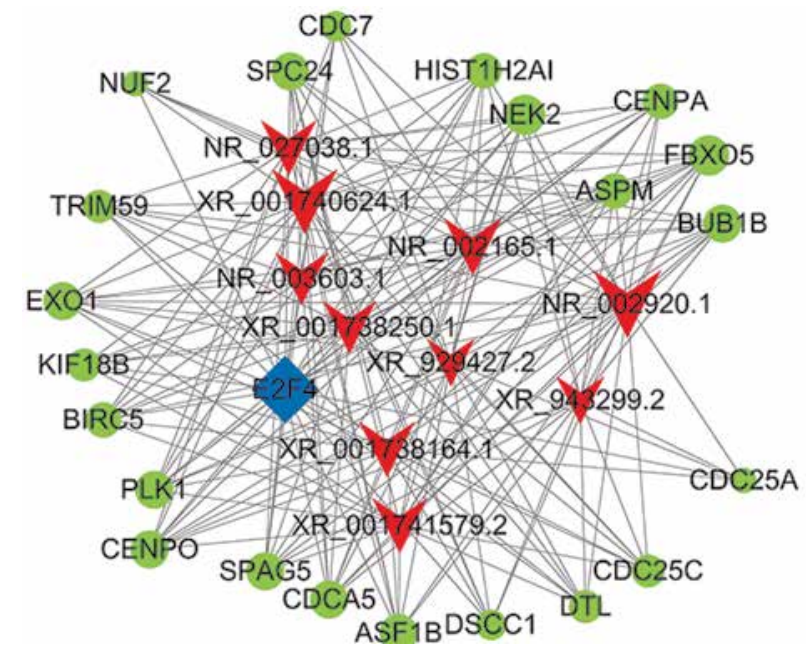

Figure 5. Prediction of trans-target for differentially expressed IncRNAs in PBM

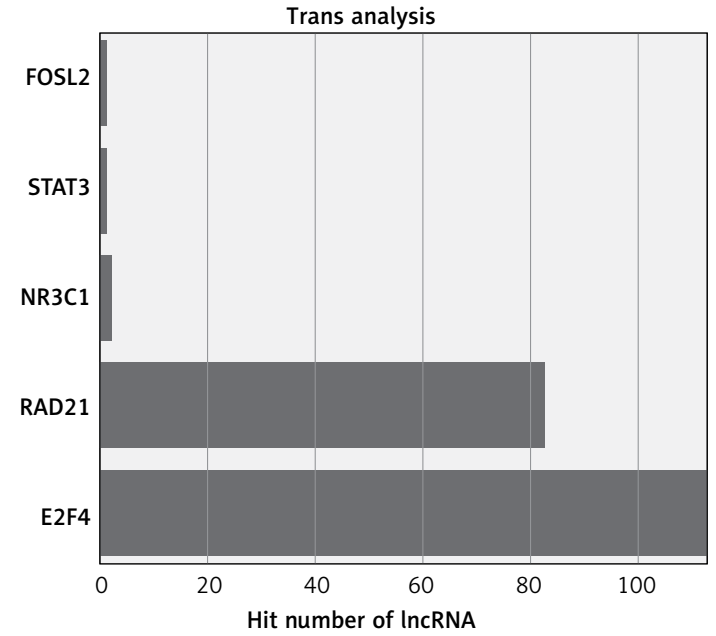

Figure 6. Functional distribution of differentially expressed IncRNAs based on transcription factor analysis
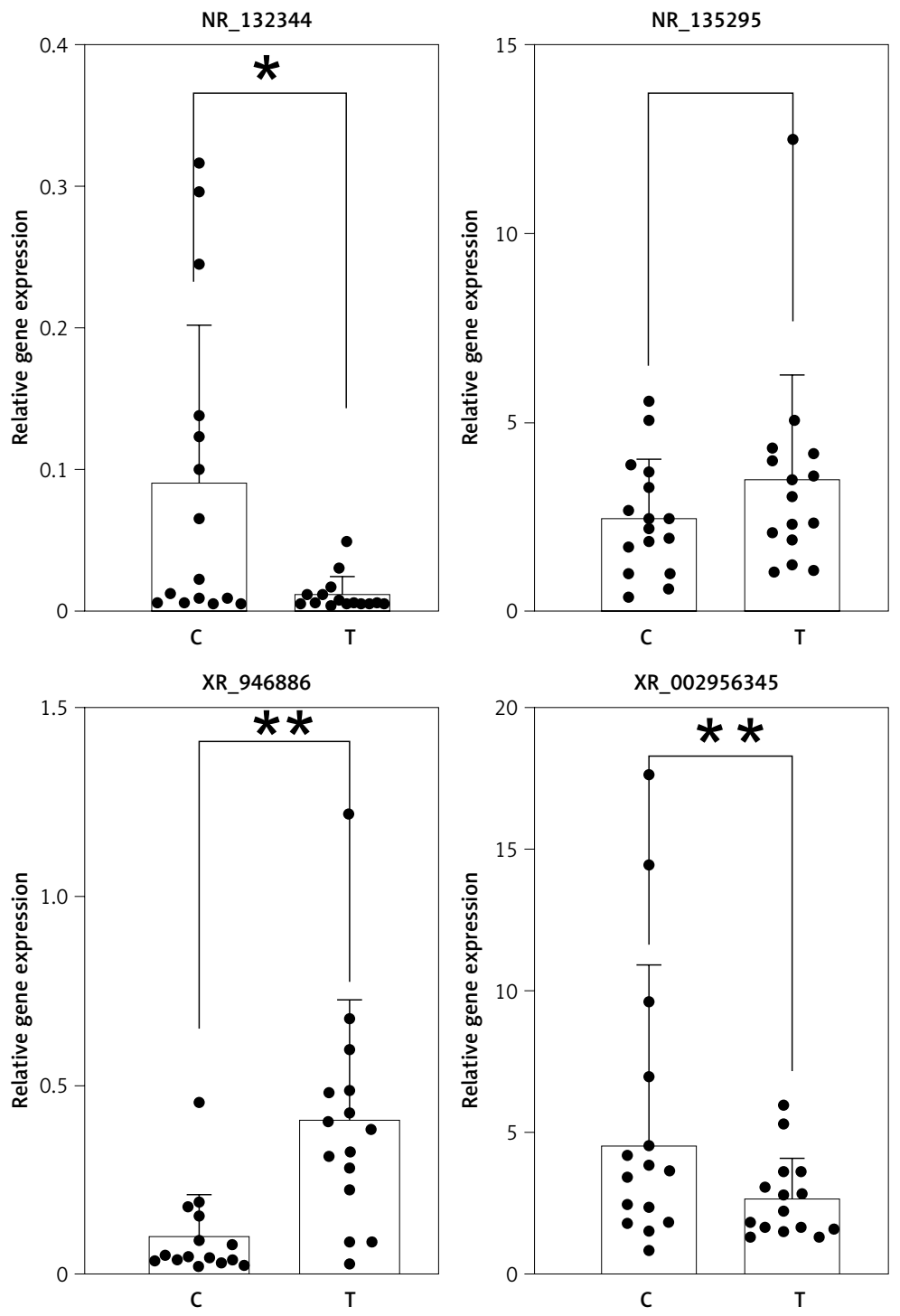

Figure 7. Validation of microarray results by $q P C R$ 


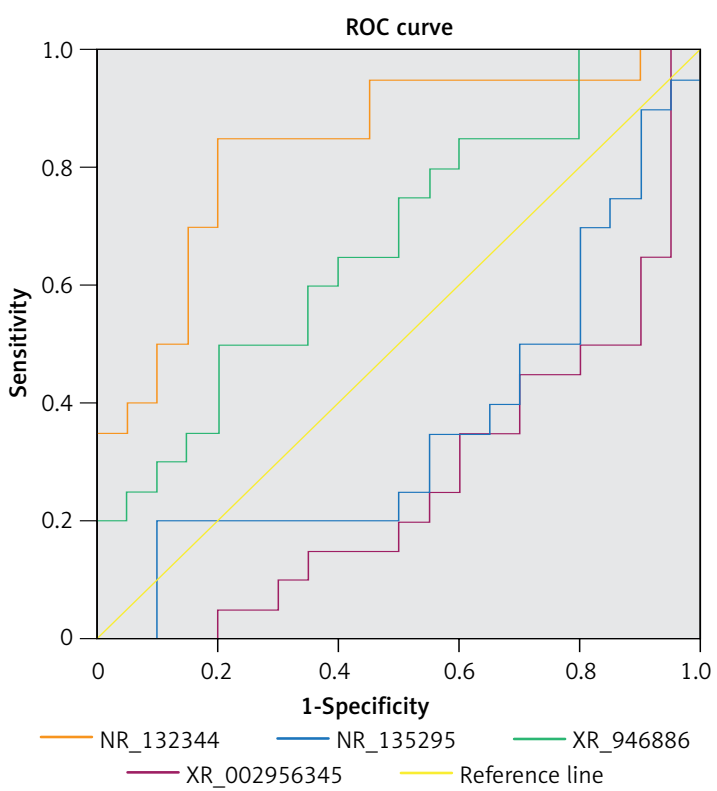

Figure 8. Receiver operating characteristic curves to assess the ability of NR_110876, NR_132344, $X R_{-}$946886, and XR_002956345 to predict pancreaticobiliary maljunction

\section{Acknowledgments}

Lian Zhao and San-Li Shi are co-first authors, they contributed equally to this work.

This work was supported by the National Natural Science Foundation of China (81971685). Scientific research project of Jiangsu Provincial Health Commission (No. ZD2022015) and Science and Technology Development Project of Suzhou (SKY2022054).

\section{Conflict of interest}

The authors declare no conflict of interest.

\section{References}

1. Le Roy B, Gagnière J, Filaire L, Fontarensky M, Hordonneau C, Buc E. Pancreaticobiliary maljunction and choledochal cysts: from embryogenesis to therapeutics aspects. Surg Radiol Anat 2016; 38: 1053-60.

2. Kamisawa T, Kuruma S, Chiba K. Congenital biliary dilatation and pancreaticobiliary maljunction. Nihon Shokakibyo Gakkai Zasshi 2016; 113: 1991-7.

3. Urushihara N, Hamada Y, Kamisawa T, Fujii H, Koshinaga T, Morotomi $\mathrm{Y}$, et al. Classification of pancreaticobiliary maljunction and clinical features in children. J Hepatobiliary Pancreat Sci 2017; 24: 449-55.

4. Kamisawa T, Kuruma S, Tabata T, Chiba K, Iwasaki S, Koizumi S, et al. Pancreaticobiliary maljunction and biliary cancer. J Gastroenterol 2015; 50: 273-9.

5. Kamisawa T, Kaneko K, Itoi T, Ando H. Pancreaticobiliary maljunction and congenital biliary dilatation. Lancet Gastroenterol Hepatol 2017; 2: 610-8.

6. Eda K, Mizuochi T, Takaki Y, Higashidate N, Hashizume N, Fukahori S, et al. Adenomyomatosis of the gallbladder with pancreaticobiliary maljunction in a child. J Pediatr Gastroenterol Nutr 2018; 67: e82.

7. Ono S, Fumino S, Iwai N. Diagnosis and treatment of pancreaticobiliary maljunction in children. Surg Today 2011; 41: 601-5.

8. Kamisawa T, Ando H, Suyama M, Shimada M, Morine $Y$, Shimada H; Working Committee of Clinical Practice Guidelines for Pancreaticobiliary Maljunction; Japanese Study Group on Pancreaticobiliary Maljunction. Japanese clinical practice guidelines for pancreaticobiliary maljunction. J Gastroenterol 2012; 47: 731-59.

9. Guo WL, Zhan Y, Fang F, Huang SG, Deng YB, Zhao JG, et al. Factors affecting the operating time for complete cyst excision and Roux-en-Y hepaticojejunostomy in paediatric cases of congenital choledochal malformation: a retrospective case study in Southeast China. BMJ Open 2018; 8: e022162.

10. Tsuchida A, Kasuya K, Endo M, Saito H, Inoue K, Nagae I, et al. High risk of bile duct carcinogenesis after primary resection of a congenital biliary dilatation. Oncol Rep 2003; 10: 1183-7.

11. Kobayashi S, Asano T, Yamasaki M, Kenmochi T, Nakagohri T, Ochiai T. Risk of bile duct carcinogenesis after excision of extrahepatic bile ducts in pancreaticobiliary maljunction. Surgery 1999; 126: 939-44.

12. Sheng Q, Lv Z, Xu W, Xiao X, Liu J, Wu Y. Reoperation after cyst excision with hepaticojejunostomy for choledochal cysts: our experience in 18 cases. Med Sci Monit 2017; 23: 1371-7.

13. Sugiura T, Endo T, Ito K, Goto K, Sato Y, Kondo S, et al. Recurrent cholangitis with congenital hepatic fibrosis and pancreaticobiliary maljunction after Roux-en-Y reconstruction. European J Pediatr Surg Rep 2013; 1: 43-5.

14. Song W, Miao DL, Chen L. Comprehensive analysis of long noncoding RNA-associated competing endogenous RNA network in cholangiocarcinoma. Biochem Biophys Res Commun 2018; 506: 1004-12.

15. Liu J, Chen L, Pan J, Chen M, Zhou J, Zhou F, et al. Comprehensive analysis of key IncRNAs in HCV-positive hepatocellular carcinoma. Arch Med Sci 2020; 17: 142-151.

16. Ren GJ, Fan XC, Liu TL, Wang SS, Zhao GH. Genome-wide analysis of differentially expressed profiles of mRNAs, InCRNAs and circRNAs during Cryptosporidium baileyi infection. BMC Genomics 2018; 19: 356.

17. Dolcino M, Pelosi A, Fiore PF, Patuzzo G, Tinazzi E, Lunardi $C$, et al. Long non-coding RNAs play a role in the pathogenesis of psoriatic arthritis by regulating microRNAs and genes involved in inflammation and metabolic syndrome. Front Immunol 2018; 9: 1533.

18. Tang Y, Zhou T, Yu X, Xue Z, Shen N. The role of long non-coding RNAs in rheumatic diseases. Nat Rev Rheumatol 2017; 13: 657-69.

19. Sallam T, Sandhu J, Tontonoz P. Long noncoding RNA discovery in cardiovascular disease: decoding form to function. Circ Res 2018; 122: 155-66.

20. Liang Y, Zhu H, Chen J, Lin W, Li B, Guo Y. Construction of relapse-related IncRNA-mediated ceRNA networks in Hodgkin lymphoma. Arch Med Sci 2020; 16: 1411-8.

21. Zhou M, Zhao H, Xu W, Bao S, Cheng L, Sun J. Discovery and validation of immune-associated long non-coding RNA biomarkers associated with clinically molecular subtype and prognosis in diffuse large B cell lymphoma. Mol Cancer 2017; 16: 16.

22. Song P, Jiang B, Liu Z, Ding J, Liu S, Guan W. A three-InCRNA expression signature associated with the prog- 
nosis of gastric cancer patients. Cancer Med 2017; 6: 1154-64.

23. Guo WL, Huang SG, Wang J, Sheng M, Fang L. Imaging findings in 75 pediatric patients with pancreaticobiliary maljunction: a retrospective case study. Pediatr Surg Int 2012; 28: 983-8.

24. Guttman M, Amit I, Garber M, French C, Lin MF, Feldser $D$, et al. Chromatin signature reveals over a thousand highly conserved large non-coding RNAs in mammals. Nature 2009; 458: 223-7.

25. Kaneko K, Ito Y, Ono Y, Tainaka T, Tsuchiya H, Shimoyama $Y$, et al. Gene expression profiling reveals upregulated UCA1 and BMF in gallbladder epithelia of children with pancreaticobiliary maljunction. J Pediatr Gastroenterol Nutr 2011; 52: 744-50.

26. Guo WL, Geng J, Zhao JG, Fang F, Huang SG , Wang J. Gene expression profiling reveals upregulated FUT1 and MYBPC1 in children with pancreaticobiliary maljunction. Braz J Med Biol Res 2019; 52: e8522.

27. Wong JK, Campbell D, Ngo ND, Yeung F, Cheng G, Tang CSM, et al. Genetic study of congenital bile-duct dilatation identifies de novo and inherited variants in functionally related genes. BMC Med Genomics 2016; 9: 75.

28. Bhawe K, Roy D. Interplay between NRF1, E2F4 and MYC transcription factors regulating common target genes contributes to cancer development and progression. Cell Oncol (Dordr) 2018; 41: 465-84.

29. Long X, Song K, Hu H, Tian Q, Wang W, Dong Q et al. Long non-coding RNA GAS5 inhibits DDP-resistance and tumor progression of epithelial ovarian cancer via GAS5-E2F4-PARP1-MAPK axis. J Exp Clin Cancer Res 2019; 38: 345.

30. Zhao L, Shi SL, Guo WL. LncRNA expression prole, functional analysis and potential biomarkers in children with pancreaticobiliary maljunction. Research square. Cell Biology 2021; 5: 25. 